\title{
Unified SPH Model for Fluid-Shell Simulations
}

\author{
Toon Lenaerts \\ Philip Dutré
}

Report CW 530, November 2008

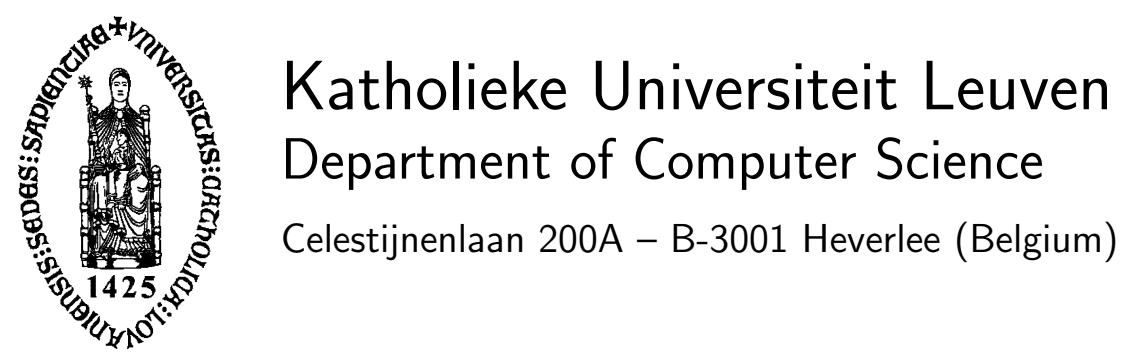




\title{
Unified SPH Model for Fluid-Shell Simulations
}

\author{
Toon Lenaerts \\ Philip Dutré
}

Report CW 530, November 2008

Department of Computer Science, K.U.Leuven

\begin{abstract}
We present the two-way coupling of a fluid to thin deformable shells in a unified particle model. We use Smoothed Particle Hydrodynamics (SPH) for the simulation of both fluid and shells. Our cloth framework extends earlier work which uses SPH to simulate deformable and rigid volumes. Our results show realistic shell and cloth animations interacting with fluids without any leaks.
\end{abstract}

Keywords : fluids, shells, deformables, smoothed particle hydrodynamics, particles.

CR Subject Classification : I.3 COMPUTER GRAPHICS, I.3.7 ThreeDimensional Graphics and Realism, Animation 


\title{
Unified SPH Model for Fluid-Shell Simulations
}

\author{
Toon Lenaerts Philip Dutré
}

November 17, 2008

\begin{abstract}
We present the two-way coupling of a fluid to thin deformable shells in a unified particle model. We use Smoothed Particle Hydrodynamics (SPH) for the simulation of both fluid and shells. Our cloth framework extends earlier work which uses SPH to simulate deformable and rigid volumes. Our results show realistic shell and cloth animations interacting with fluids without any leaks.
\end{abstract}

\section{Introduction}

In recent years, animations featuring fluid interactions are modelled more and more using simulation algorithms. Particle-based simulation methods can cope with different kinds of objects and materials, such as fluids or deformable bodies, in a natural way.

One of the most popular particle methods for simulating fluid flow coupled to other bodies is Smoothed Particle Hydrodynamics (SPH). However, coupling thin shells, consisting only of one layer of particles, and fluids provides a challenge in avoiding leaking behavior.

In this work we extend previous work on interactions between fluids and deformable bodies to interactions between fluids and thin elastic shells and cloth in one unified SPH framework. We employ an explicit collision handling scheme which couples fluids to thin elastic shells without leaks.

In the next sections we only illustrate how previous work can be extended to incorporate these new simulation effects. We refer to the original papers for a good understanding of the overall particle framework.

\section{Fluid-Shell Simulation}

Our fluid framework is based on the work of Müller et al. [3], which uses SPH to solve the Navier-Stokes momentum equation for fluid flow. We refer to the work of Monaghan [2] for a good overview on SPH. Particles are integrated in time using the leap-frog integration scheme.

Solenthaler et al. [5] use SPH for its ability to handle coarsely and coplanar sampled particles and uses it for melting and solidification simulations. This motivates us to extend and apply their elastic model to thin shells. Only relying on fluid pressure and 
elastic forces as in [5] is not enough to overcome leaks as high fluid pressures or low Young's moduli may lead to higher spacings between particles. Therefore, we add an explicit collision handling scheme based on the molecular dynamics approach of Bell et al. [1] (Figure 2).

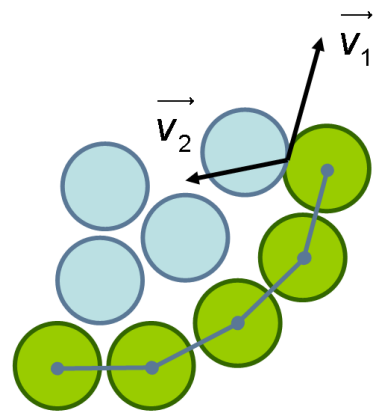

Figure 1: Explicit collision handling between fluid particles (blue) and shell particles (green) is added based on the molecular dynamics approach of Bell et al. [1].

\section{Cloth Simulation}

We extend the elastic model of Solenthaler et al. [5] to allow the simulation of cloth. They compute elastic forces for each particle using a local reference neighborhood in which each particle stores distance vectors to its neighboring particles in rest (see Figure 3). Displacements from this reference neighborhood translate into a strain $\epsilon$ from which the stress $\sigma$ can be computed using Hooke's law, $\sigma=\mathbf{C} \epsilon$. The extension for cloth simulation is achieved by discarding the orientation of particles in the reference neighborhood and using only the distance between neighboring particles. Doing so appropriately allows for bending, but prohibits material stretching.

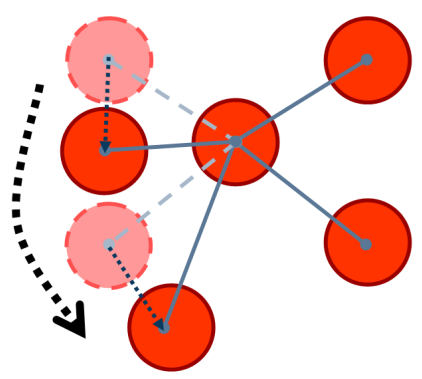

Figure 2: Each elastic particle stores a reference neighborhood (dashed particles). Displacements from this reference neighborhood translate into a strain $\epsilon$ from which the stress $\sigma$ can be computed using Hooke's law. Only accounting for the distances between neighboring particles, allows for bending, but prohibits material stretching.

Though the elastic model tries to keep a minimum distance between particles in the reference neighborhood, self-collisions can still occur when pieces of cloth are folded and overlap. We avoid this by applying a simple penalty force proportional to the overlap between particles, hereby pushing the particles away from eachother. 


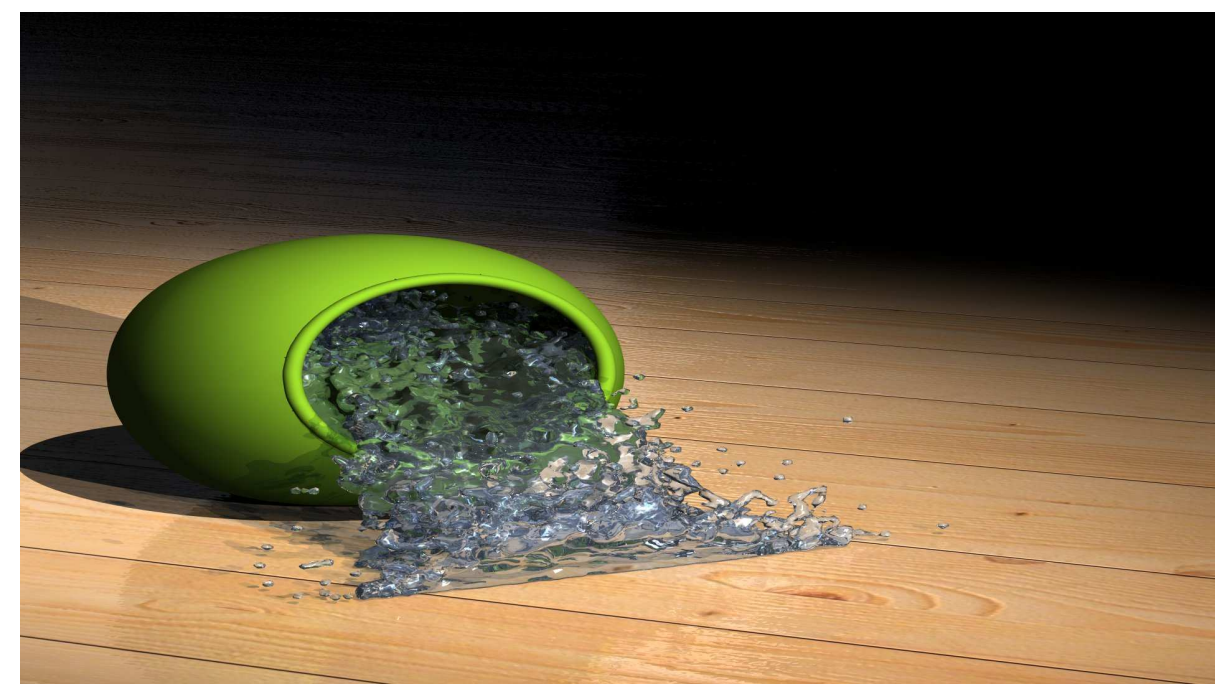

Figure 3: An elastic bowl filled with water is dropped on the floor. The bowl deforms on impact but water only splashes out of the bowl through the side opening.

Particles attached to a fixed point such as the cloth in Figure 5 or elastic particles colliding with fluid can experience a lot of stress. The Rayleigh damping scheme is used damp and stabilize the cloth, without damping the overall motion of the cloth.

\section{Surface Animation}

The surface of the shell in our simulation framework is defined by a triangle mesh that is animated along with the simulation particles. Neighboring particles are computed for each vertex in the object's undeformed rest shape. The deformation of each vertex can then be efficiently computed from the neighboring particles' deformations using the embedded deformation approach [4]. The surfaces of the fluid particle volumes are extracted using Marching Cubes as in Solenthaler et al. [5] and are rendered in POV-Ray.

\section{Results and Discussion}

Our simulations are rendered as animations which can be seen in the accompanying video.

Figure 3 shows a thin elastic bowl filled with water. The bowl is dropped on the floor which causes it to deform on impact. During the impact the water further deforms the bowl, however, without any leaks. Water only splashes through the side opening out of the bowl. The bowl consist out of 5,300 particles and contains 48,000 water particles.

A piece of cloth is dropped over 5 floating spheres in Figure 4. Then, water is poured on the cloth which wraps around the raising water mass until it slips through the spheres 


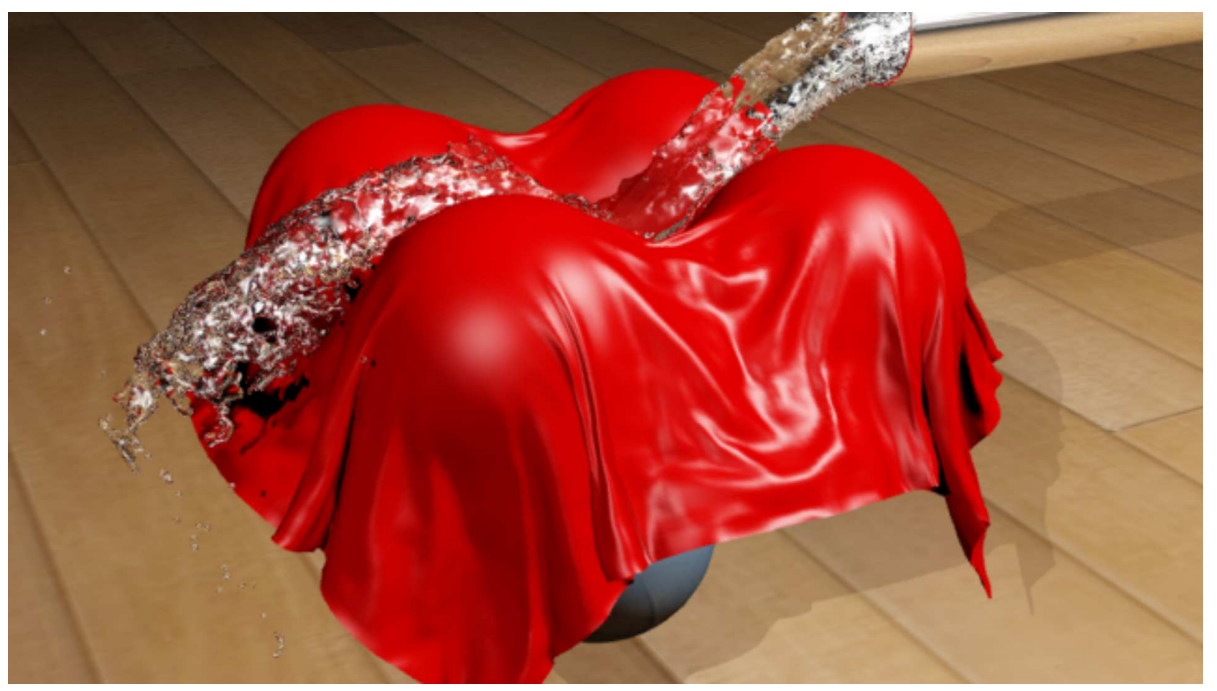

Figure 4: A piece of cloth is dropped over floating spheres. Water is then poured on the cloth which slips through the sphere as the water mass increases.

above. The cloth is simulated using 15,000 particles. For the water simulation about 100,000 particles were used.

In Figure 5 a jet of water interacts with a piece of cloth. Notice how the force of the water jet pushes the cloth away, after which the cloth sweeps back again and influences the water flow. The water is simulated using 325,000 particles while the cloth simulation only uses 15,000 particles.

While the collision scheme of Bell et al. [1] provides a good coupling between fluid and thin shell, there still are circumstances under which water leaks can occur. For large shell deformations fluid particles can still leak between stretched elastic particles. In such situations, a resampling of the elastic shell could solve the leaks. However, we believe our method is sufficient for most animations, as the more extreme bowl example (Figure 3) illustrates.

\section{Conclusion}

We showed how the framework of Solenthaler et al. [5] can be extended to allow thin shell simulations in an SPH fluid environment using explicit collision handling. Furthermore, by altering their local reference shape definition, we are able to perform SPH cloth simulations. Our animations show fluid-shell interactions without leaks.

Acknowledgements Toon Lenaerts is funded by a Ph.D. grant of the Institute for the Promotion of Innovation through Science and Technology in Flanders (IWT-Vlaanderen). 


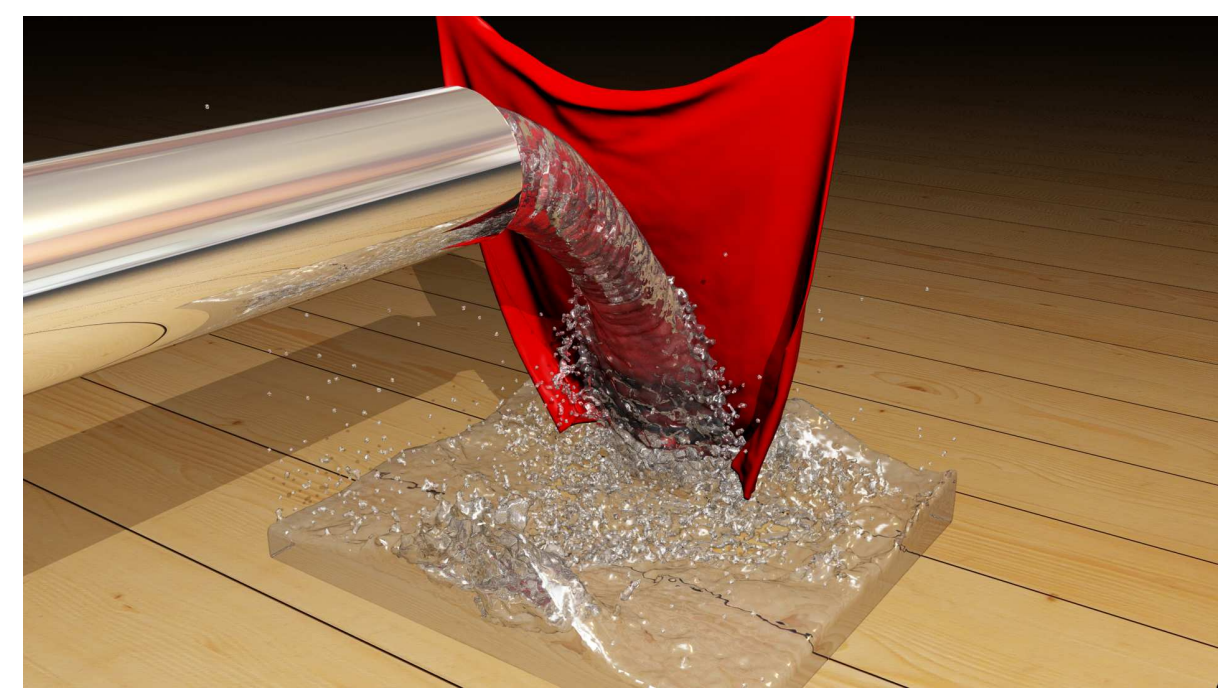

Figure 5: A jet of water is aimed at a piece of cloth. Water slides of the cloth which sweeps back and forth by the interplay of the jet.

\section{References}

[1] Nathan Bell, Yizhou Yu, and Peter J. Mucha. Particle-based simulation of granular materials. In SCA '05: Proceedings of the 2005 ACM SIGGRAPH/Eurographics symposium on Computer animation, pages 77-86, New York, NY, USA, 2005. ACM Press.

[2] J. J. Monaghan. Smoothed particle hydrodynamics. Reports on Progress in Physics, 68(8):1703-1759, August 2005.

[3] Matthias Müller, David Charypar, and Markus Gross. Particle-based fluid simulation for interactive applications. In SCA '03: Proceedings of the 2003 ACM SIGGRAPH/Eurographics symposium on Computer animation, pages 154-159, Airela-Ville, Switzerland, Switzerland, 2003. Eurographics Association.

[4] Matthias Müller, R. Keiser, A. Nealen, M. Pauly, M. Gross, and M. Alexa. Point based animation of elastic, plastic and melting objects. In SCA '04: Proceedings of the 2004 ACM SIGGRAPH/Eurographics symposium on Computer animation, pages 141-151, Aire-la-Ville, Switzerland, Switzerland, 2004. Eurographics Association.

[5] Barbara Solenthaler, Jürg Schläfli, and Renato Pajarola. A unified particle model for fluid-solid interactions: Research articles. Comput. Animat. Virtual Worlds, 18(1):69-82, 2007. 